\title{
An Efficient Image Mosaic Algorithm Based on EMD Transform
}

\author{
Yi-jun Wang ${ }^{1, a)}$ and Sen Wei ${ }^{1, b)}$ \\ ${ }^{1}$ Institute of Information Science and Engineering, Central South University, Changsha 410075, \\ China. \\ a)522134654@qq.com, ${ }^{\text {b) }}$ csu_weisen@163.com
}

Keywords. empirical mode decomposition (EMD), image compression, image mosaic, Harris algorithm.

\begin{abstract}
This paper proposes an image mosaic algorithm based on empirical mode decomposition (EMD) transform. A complete, fast and efficient EMD image decomposition algorithm is used to decompose the image, and the inverse discrete cosine transform (IDCT) is performed on the result of the transformation. After the interpolation operation, the compressed image is obtained. Then, the Harris algorithm is used to extract the feature points and match these points. The accuracy of the feature point registration is improved by using the triangular area of the first and second adjacent points, and the EMD algorithm is used to compress the image, which greatly reduces the time of image splicing.
\end{abstract}

\section{I.INTRODUCTION}

At present, there are a lot of researches on the key technology of image stitching, image matching and image fusion. However most of the methods in the complex scene image splicing effect is not ideal, and high precision stitching is slowly, time-consuming, poor real-time and can not meet the requirements of fast stitching. The main method of image matching is using C. Harris's Harris operator to detect the feature points [1]. Not only the detection speed, but also has the rotation and translation invariance, can achieve sub-pixel level accuracy, and have good robustness.

The empirical model is decomposed with a new method for nonlinear and unstable data analysis proposed by NASA's Norden E. Huang in 1998 [2]. The two-dimensional empirical mode decomposition has the ability to decompose the image into local narrowband signals. It is born to be used in image processing. At present, there are a variety of attempts base on EMD method in the field of image research [3-6]. In this paper, an image mosaic algorithm based on empirical mode decomposition is presented. The image is subjected to EMD compression processing, and then Harris algorithm is used to stitch the image, and the triangular area of the nearest neighbor feature points is used to remove the pseudo-feature points. To achieve fast, high-precision image stitching.

\section{IMAGE MOSAIC ALGORITHM BASED ON EMD TRANSFORM}

\section{A. IMAGE DECOMPOSITION BY EMD ALGORITHM}

The image is decomposed by EMD image decomposition algorithm. After the image is processed by empirical mode decomposition (EMD), the Intrinsic Mode Function (IMF) image and the remaining image can be obtained. The EMD decomposition method of the two-dimensional image F can be carried out as follows:

1) Find the local extreme point of plane $F$ by 8 neighborhoods.

2) The triangular subdivision of the plane $f$ extreme point is prepared for the next interpolation.

3) On the triangular mesh, the interpolated surfaces $e_{\max }$ and $e_{\text {mix }}$ are constructed according to the extreme points, and the algebraic mean of the maximal envelope surface $e_{\max }$ and the minimum envelope surface is ave $=\frac{1}{2}\left(e_{\max }+e_{\text {mix }}\right)$.

4) Subtract the mean ave from the remaining image plane $F$ data to get the margin $\mathrm{h}=\mathrm{f}-$ ave. 
5) The margin $h$ is used as the new data value, repeat steps (1) to (4) until h satisfies the end condition. At this time, $\mathrm{h}$ is regarded as an inherent mode function-imf.

6) Find the first inherent mode function $i m f_{1}$, and then the remaining item rem as a new data to continue in accordance with the above steps in order to extract a number of inherent mode function $i m f_{n}$.

In the general literature, the median value of the maximum and minimum envelope is less than a certain threshold as the end condition:

$$
\left|e_{\operatorname{mid}(i, j)}\right|<\varepsilon
$$

According to the end condition of the formula (1), although the decomposition result can be obtained, the image decomposition speed is slow, the number of layers is large, and the difference between the layer and the layer is large. The recursive residual image is presented using the gray scale mean in paper[7]:

$$
f_{\text {ave }}=\frac{1}{m n} \sum_{i=1}^{m} \sum_{j=1}^{n} f(i, j)
$$

Using the curve curvature is less than a certain threshold $\varepsilon$ as the end condition, the image is decomposed to improve the efficiency of decomposition.

\section{B. IMAGE COMPRESSION BY IDCT ALGORITHM}

According to the EMD image decomposition improvement algorithm proposed above, the image is decomposed by one layer to obtain an intrinsic mode function image and the residual image, the native model function has the detail information (texture) of the original image, and the gray scale distribution changes violently. The residual image has the trend information of the original image and the gray scale distribution is flat. So we can make full use of the decomposition results, take a different compression strategy for the inherent mode function image and the remaining image compression. In this paper, we take the method of sampling and adding discrete cosine transform to the image of the inherent mode function, and take the discrete cosine transform method for the residual image. The specific processing steps are as follows [8-10]:

1) The original image is decomposed by EMD decomposition algorithm.

2) The inherent mode function image and the remaining image are divided into $7 \times 7$ small blocks, and the edges of the image blocks of the inherent mode function are overlapped by one line or one column.

3) The inherent mode function image is sampled in small pieces and the sample is subjected to discrete cosine transform to extract the transformation result exceeding the set threshold $\varepsilon_{1}$. The discrete cosine transform is directly performed on the small pieces of the remaining images to extract the partial values of the transformation result $\{F(i, j), i, j=1,2, \wedge, 7\}$.

4) Inverse discrete cosine transform (IDCT) is performed according to the extracted transform result. The small pieces of the image of the intrinsic mode function are also subjected to the surface interpolation operation according to the IDCT result.

5) The inherent mode function image and the remaining image obtained by IDCT are superimposed to obtain the decompressed image.

\section{AN IMPROVED IMAGE MOSAIC ALGORITHM BASED ON HARRIS}

After the image is compressed by the above steps, the Harris operator is used to extract the feature points of the mosaic image, and then the feature points are registered with the Cross-Correlation theory. And then use the triangular area of the feature point of the first and second adjacent point pairs to remove the pseudo-registration, and the correct registration point is found. Stitching, and finally using the wavelet transform for image fusion to achieve image stitching. Specific steps are as follows:

1) Feature point extraction. Using Harris algorithm to extract features points of the two image to be spliced:

Among them

$$
R=\operatorname{det}(A)-k \cdot \operatorname{tr}^{2}(\mathrm{~A})
$$




$$
\mathrm{A}(\mathrm{i}, \mathrm{j},)=\mathrm{W}\left[\begin{array}{ll}
I_{x}^{2}(i, j) & I_{x y}(i, j) \\
I_{x y}(i, j) & I_{x}^{2}(i, j)
\end{array}\right]
$$

Where $\mathrm{W}$ is the Gaussian smoothing window, $\mathrm{k}$ is the empirical value, and if $\mathrm{R}$ is greater than the given threshold $\mathrm{T}$, it is the feature point.

2) Feature point registration. The feature points are registered with the Cross-Correlation theory.Take a $(2 \mathrm{~N}+1) \times(2 \mathrm{~N}+1)$ correlation window with each feature point as the center to calculate the correlation coefficient between the correlation points of the feature points:

$$
\mathrm{R}=\frac{\sum_{i=-N}^{N} \sum_{j=-N}^{N}(I(x-i, y-j)-\bar{I})\left(I^{\prime}\left(x^{\prime}-i, y^{\prime}-j\right)-\bar{I}^{\prime}\right)}{\sqrt{\sum_{i=-N}^{N} \sum_{j=-N}^{N}(I(x-i, y-j)-\bar{I})^{2} \sum_{i=-N}^{N} \sum_{j=-N}^{N}\left(I^{\prime}\left(x^{\prime}-i, y^{\prime}-j\right)-\bar{I}^{\prime}\right)^{2}}}
$$

3) Remove the pseudo-registration points. In this paper, we use triangular area of the feature point of the first and second adjacent point pairs to remove the pseudo-registration. Suppose that $\mathrm{A}\left(x_{a}, y_{a}\right), A^{\prime}\left(x_{a}^{\prime}, y_{a}^{\prime}\right)$ is a pair of feature points, $\mathrm{B}\left(x_{b}, y_{b}\right), B^{\prime}\left(x_{b}^{\prime}, y_{b}^{\prime}\right)$ and $\mathrm{C}\left(x_{c}, y_{c}\right), C^{\prime}\left(x_{c}^{\prime}, y_{c}^{\prime}\right)$ is its first and second adjacent feature point pairs, then the European distance of the three feature points in the first picture as follow

$$
\begin{gathered}
\mathrm{a}=\sqrt{\left(x_{a}-x_{b}\right)^{2}+\left(y_{a}-y_{b}\right)^{2}} \\
\mathrm{~b}=\sqrt{\left(x_{b}-x_{c}\right)^{2}+\left(y_{b}-y_{c}\right)^{2}} \\
\mathrm{c}=\sqrt{\left(x_{c}-x_{a}\right)^{2}+\left(y_{c}-y_{a}\right)^{2}}
\end{gathered}
$$

The area of they constituted

$$
\mathrm{S}=\frac{1}{4} \sqrt{(a+b+c)(a+b-c)(a+c-b)(b+c-a)}
$$

Similarly, the area of the second image can be obtained. It is recorded as $S^{\prime}$.If the following relationship is satisfied, they can be judged as correct pairs of features.

$$
\mathrm{S}=\mathrm{S}^{\prime}
$$

Estimation the parameter of transformation matrix. After finding the correct registration point, the RANSAC algorithm is used to calculate the perspective transformation model for image splicing [11].

4) Image fusion. Wavelet transform is used in image fusion on the spliced image in order to achieve good visual effect [12].

\section{EXPERIMENTAL VALIDATION}

In order to verify the above theory, the use of EMD algorithm to compress the image as shown in figure 1.
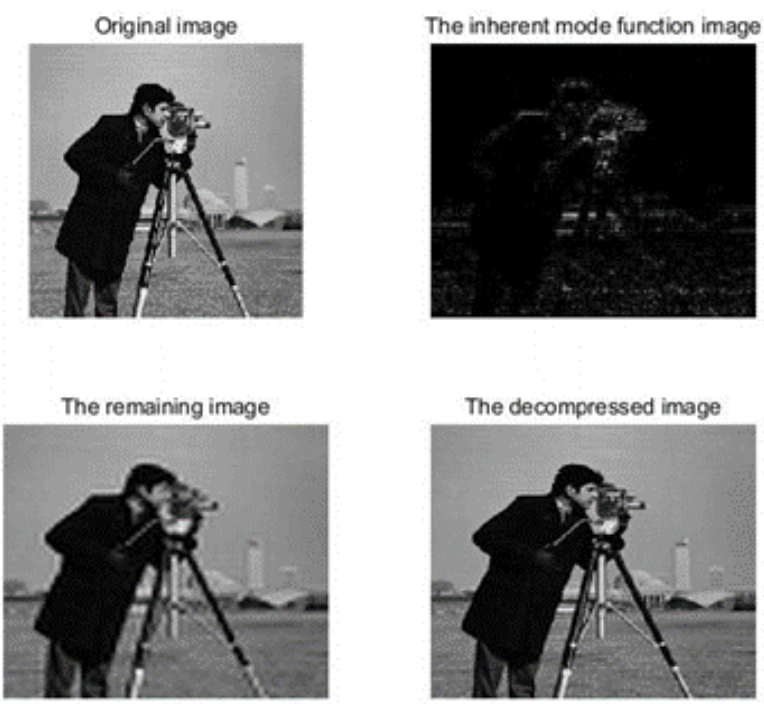

FIGURE 1. The decompression of image by the EMD 
The results show that the algorithm can obtain a higher compression peak signal to noise ratio (PSNR $=28.1546 \mathrm{~dB}$, bpp $=0.5431)$ with lower bits, and the decompression visual effects is good.

The splicing algorithm proposed in this paper is used to splice the two images, which are processed by EMD. The experimental results are as follows

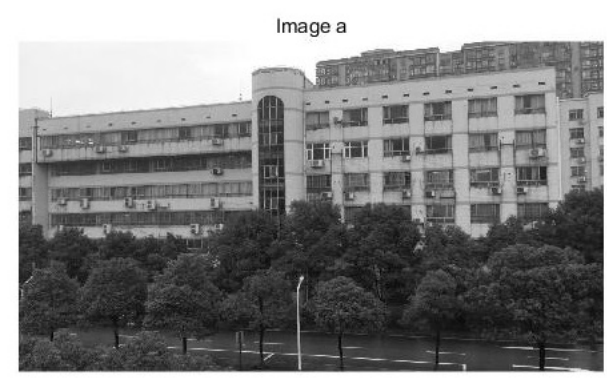

(a)

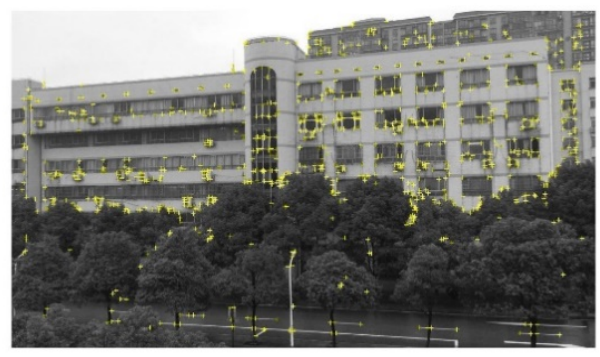

(c)

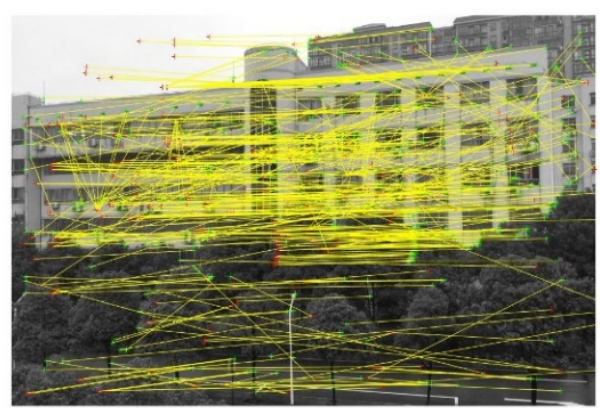

(e)

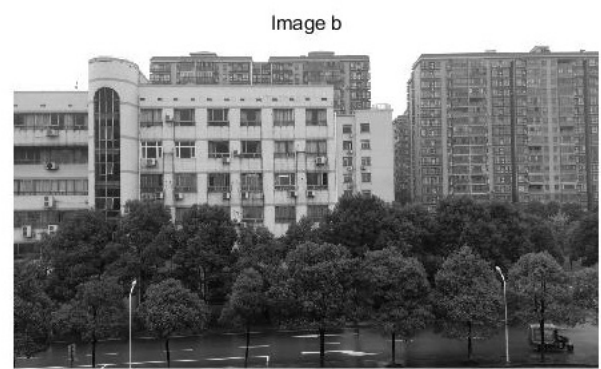

(b)

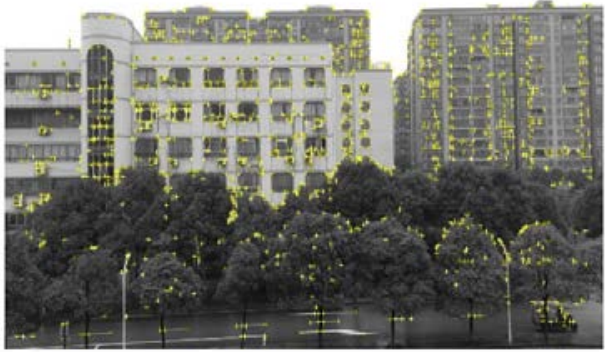

(d)

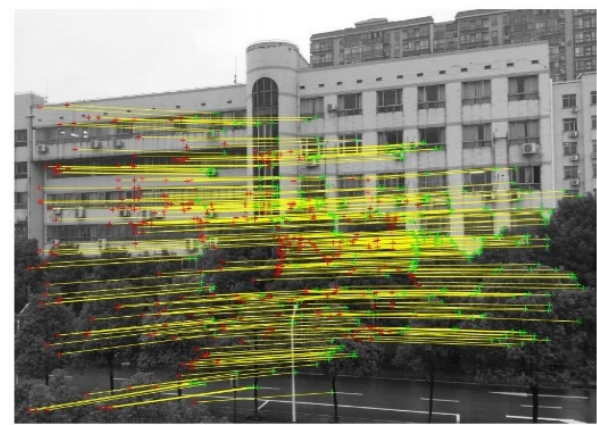

(f)

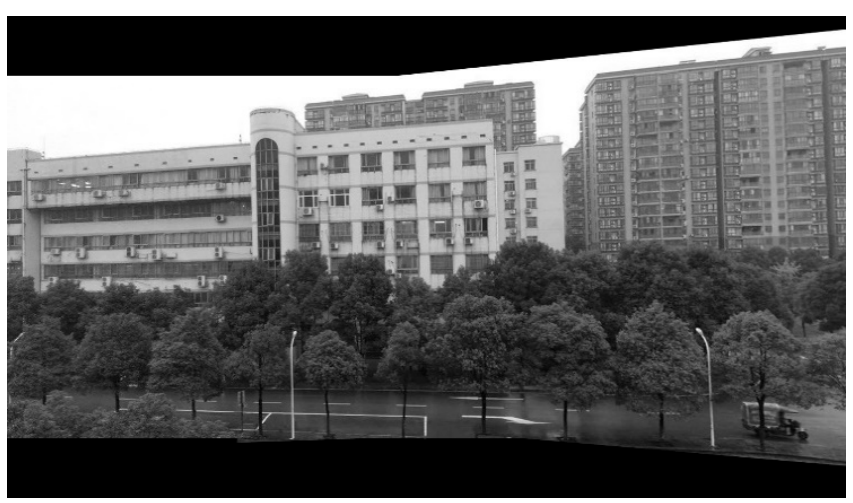

(g)

FIGURE 2. The image mosaic algorithm proposed in this paper

In figure 2, (a),(b) are the two images to be spliced;(c),(d) is the two images using Harris operator to extract the feature points;(e) is the feature point pairs using Cross-Correlation theory showed in the image a;(f) shows that the feature point pairs after remove the pseudo-registration points; $(\mathrm{g})$ is the effect of splicing using the algorithm described in this paper.

At the same time using the original algorithm for image splicing, compared with the algorithm proposed in this paper, as shown in Table 1. 
TABLE 1. Comparison of two methods

\begin{tabular}{ccccc}
\hline Methods & $\begin{array}{c}\text { Coarse } \\
\text { match }\end{array}$ & $\begin{array}{c}\text { Fine } \\
\text { match }\end{array}$ & $\begin{array}{c}\text { Remove false } \\
\text { registration }\end{array}$ & Time \\
\hline $\begin{array}{c}\text { Original } \\
\text { algorithm }\end{array}$ & 256 & 193 & 63 & $4.715 \mathrm{~s}$ \\
\hline $\begin{array}{c}\text { Proposed } \\
\text { algorithm }\end{array}$ & 256 & 121 & 135 & $1.875 \mathrm{~s}$ \\
\hline
\end{tabular}

\section{IV.CONCLUSION}

From the experimental results, we can find that the image splicing method based on EMD transform can effectively reduce the splicing time and improve the efficiency of the algorithm. In the registration of feature points, we introduce the area of the feature points to eliminate the pseudo-registration points. Improve the accuracy of registration, and improve the stability of the transformation matrix.

\section{ACKNOWLEDGMENTS}

I want to take this chance to thank my tutor-Yi-jun Wang, a professor of Institute of Information Science and Engineering of Central South University. In the process of writing this paper, he has given me many constructive academic recommendations, and helped me to correct my paper. Except for these, he also gave me the opportunity to practice. The corresponding author of this paper is me, Sen Wei.

\section{REFERENCES}

[1] Harris C.G, Stephens MJ.A combined corner and edge detector. Processing Fourth Alvey Vision Conferece.Manchester,U.K.1988.147-151.

[2] Huang N E, Shen Z, Long S R, et a1.The empirical mode decomposition and the Hilbert spectrum for nonlinear and nonstationary time series analysis[J] .Proc. Roy. Soc. Lond A,1998,454:903-995.

[3] Rilling G, Flandfin P, Goncaires P. Empirical mode decomposition as a filter bank[J].IEEE Signal Process Lett.2004,11(2):112-114.

[4] SONG Ping-Jian, ZHANG Jie. The application of two-dimensional EMD to separating contents of oceanic remote sensing images[J].High Technology Letters,2001,11(9):62-67.

[5] Nunes J C, Bonaoune Y, DelecheUe E, et a1.Image analysis by bidimensional empirical mode decomposition[J].Image Vis.Comput,2003,21(12):1019-1026.

[6] Linderhed A.2D empirical mode decomposition in the spirit of image compression J I. Proc. SPIE, Wavelet lndependent Component Analysis Application IX,2002,4738:1-8.

[7] He Jingbo, Peng Fuyuan. An improved algorithm for EMD image decomposition[J]. High Technology Letters,2007,11(17):1121-1124.

[8] Ji Xiuhual, Zhang Caiming. A Fast IDCT Algorithm for Image Decompression[J]. Chinese Journal of Computers,2005,28(12):2079-2086.

[9] Liang Jie. Fast multiplierless approximations of the DCT with the lifting scheme[J].IEEE Transactions on Signal Processing,2001,49(12):3032-3044.

[10]Henning R. , Chakrabarti C..A quality/energy tradeoff approach for IDCT computation in MPEG-2 video decoding[J].In: Proceedings of IEEE Signal Processing Systems(SiPS),Lafayette,2000.90-99.

[11]Zhang Zhichun, Kuang Liqun, Han Xie, Yang Xiaowen. An Improved RANSAC Algorithm for Image Mosaic[J].Computer Measurement \& Control,2014,22(6):1856-1858

[12]Liu Guixi,Yang Wanhai. A Wavelet-Decomposition-Based Image Fusion Scheme and Its Performmance Evaluation[J]. Acta Automatica Sinica,2002,28(6):927-934. 